\title{
Application of an automated aircraft architecture generation and analysis tool to unmanned aerial vehicle subsystem design
}

\author{
David Judt, Craig Lawson
}

School of Engineering, Cranfield University, Cranfield, UK

\begin{abstract}
The work presents the application of a new computational framework, addressing future preliminary design needs for aircraft subsystems. The ability to investigate multiple candidate technologies forming subsystem architectures is enabled with the provision of automated architecture generation, analysis and optimisation. The core aspects involve a functional decomposition, coupled with a synergistic mission performance analysis on the aircraft, architecture and component level. This may be followed by a complete enumeration of architectures combined with a user-defined technology filtering and concept ranking procedure. In addition, a novel hybrid heuristic optimiser, based on ant colony optimisation and a genetic algorithm, is employed to produce optimal architectures in both component composition and design parameters. The framework is applied to the design of a regenerative energy system for a long endurance high altitude unmanned aerial vehicle, considering various emerging technologies. A comparison with the traditional design processes and certification requirements is made as well as technology trends summarised and substantiated.
\end{abstract}

\section{Introduction}

Aircraft conceptual design commonly relies on a database of design parameters from past vehicles when analysing new aircraft concepts [1-3]. By establishing trend lines for important variables such as thrust-to weight ratio or wing loading, a design to meet new mission requirements can be sized quickly with relatively good certainty. Utilising these past concepts and relying on proven technologies, reduces the risk associated with the project and improves the operational safety. Each evolved aircraft benefits from its predecessors design knowledge and could bring performance improvements, such as reduced fuel burn and structural mass.

However, this conventionalism also avoids advancement of revolutionary concepts, and as a result produces the very familiar configuration of civil transport aircraft operating over the past five decades. Similarly in subsystem design for civil aircraft, relying on such historical and empirical relationships significantly limits the design space to existing and welldocumented technologies. If one wishes to venture away from the conventional, common practice utilises 'fudge factors' to adjust empirical trend lines for designs which maintain their architecture to a certain degree. In the more recent field of subsystem design for uninhabited air vehicles, novel architectures are more common since the removal of safety considerations for human occupancy opens up the design space.

In the initial phases of a design cycle engineers face the challenge, where very little is known about the problem but full design freedom is available. Here, the main focus should be on exploring many potential solutions and concepts, while keeping them as changeable as possible. As concepts mature and knowledge is gained from analysis and modelling the commitment to these designs grows. An increasing resource allocation takes place as the process advances, making it costly to implement major design changes. Figure 1 demonstrates such trends for current and future design processes. 


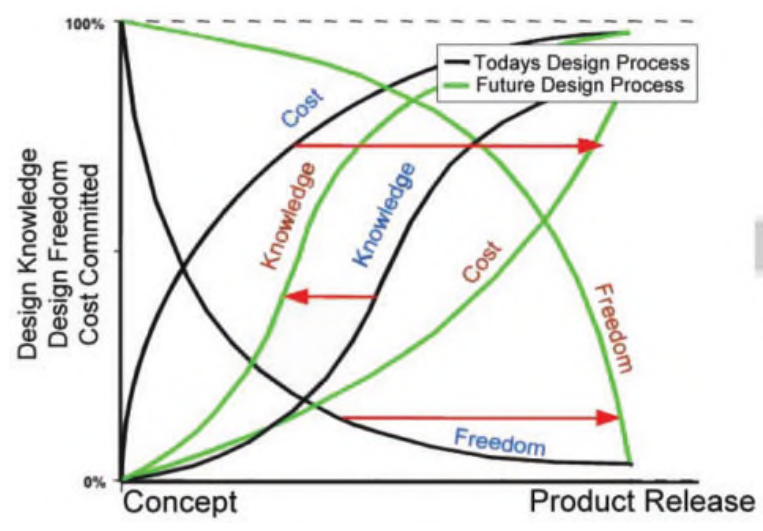

Future processes

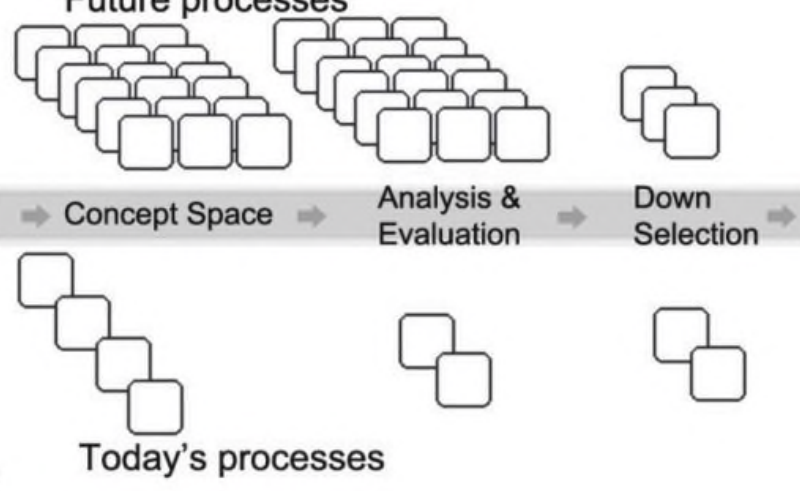

Figure 1: Current and future design processes

Increased knowledge about concepts and maintaining design options open delays the rise of committed cost during the design process. This requires flexible enough design methods to deal with a multitude of potential solutions. Another challenge is to provide a framework which has low development time requirements when applied to a new design problem. In aircraft subsystem conceptual design, there is generally more information available about the aircraft concept since the subsystem design cycle usually initiates later within the overall aircraft design process. First estimates of subsystem design metrics, such as mass and power requirements are often based on empirical equations established from design experience and past design data. [4,5] The underlying assumption implicit in the use of such a model is that the subsystem's architecture will be similar to past designs. This substantial restriction on the design freedom does not usually allow for the exploration of architectures which include novel technology components and different structural configurations. Hence in the spirit of pushing trends, as shown in Figure 1, a change in design approach seems inevitable. Ideally one would like to be able to follow similar developments as aircraft design took with multi-disciplinary optimisation methods and apply them to conceptual subsystem design. New approaches are required, due to the difficult task of generalising subsystems to represent a wide concept space with not only varying parameters but also different architectures.

One technique for facilitating this generalised formation of architectures is achieved by a functional decomposition of the design problem. [6-8] These functional requirements are generally enough to be linked to various physical component models making up the architecture concept. Combined with an intelligent framework of component filtering, functional reconfiguration and input/output variable linkages, such a process is flexibility enough to consider a diverse range of architecture types. [9] Various approaches focus on moving away from empirical methods and towards subsystem concept simulations for such an extended design space. One approach builds parametric disciplinary models for the array of aircraft subsystems. [10,11] Using expert knowledge and physical relationships, power consumption, transformation, distribution as well as generation systems can be modelled for both sizing and performance execution. Other approaches looked at automating the setup of the systems architecture model from individual component elements.[12-15] Both approaches also incorporate a feedback between subsystem sizing and the aircraft level performance. This makes it possible to study and optimise subsystems for both aircraft and subsystem level objectives.

Combining such new architecture flexible methods with gradient-based optimiser methods can be a challenge, since the cost function is often nonlinear and discrete as the architecture analysis model structure changes. Successful approaches use heuristic-based optimisers, such as genetic algorithms (GAs) and ant colonisation optimisation (ACO).[16-18] 


\section{Framework formulations}

The established framework integrates elements from past authors, such as functional induction, [6,8] component sizing, 7 flexible mission profiles [10,11] and architecture enumeration. [19] In addition, new aspects are integrated such as the component model coordination strategies, and the heuristic optimisation approach. With this collection of methods a contribution to design approaches is intended by providing higher flexibility in framework deployment, as well as a broader design space investigation. An overview of the framework is presented in Figure 2. Initially, information is required to accurately define the system design problem and its setting in the overall aircraft concept. This establishes a clear boundary between elements considered part of the subsystem and other aircraft elements. Requirements, both functional and performance are extracted and associated to component models and operational scenarios.

The follow-on analysis process requires the provision of models of individual architecture components, aircraft level performance and the external aircraft environment. Initial execution of the aircraft and external level models leads to a mission profile time series of input and output $(\mathrm{I} / \mathrm{O})$ conditions to the subsystem architectures.

Hence during each of these $1 / O$ steps, the relevant subsystem component models are executed with their connectivity information known, to provide a balanced inter-component variable flow satisfying the architecture interface $\mathrm{I} / \mathrm{O}$ conditions. Furthermore, components are sized to user-defined targets of architecture and aircraft performance, by step-wise parametric alterations with a root-finding routine. Finally, the initial architecture parameters relevant to the aircraft level are updated with the new estimates after successful sizing, leading to a repetition of the complete cycle. For a pure enumeration, this process is repeated for all unique architectures from the function tree. Such an enumeration is fully automated with a new function tree level-based morphological matrix method and userdefined combination filters. The function tree can also be limited, to produce just certain architectures, then undergoing a parametric grid search to investigate their response to aircraft, mission or component design parameters.

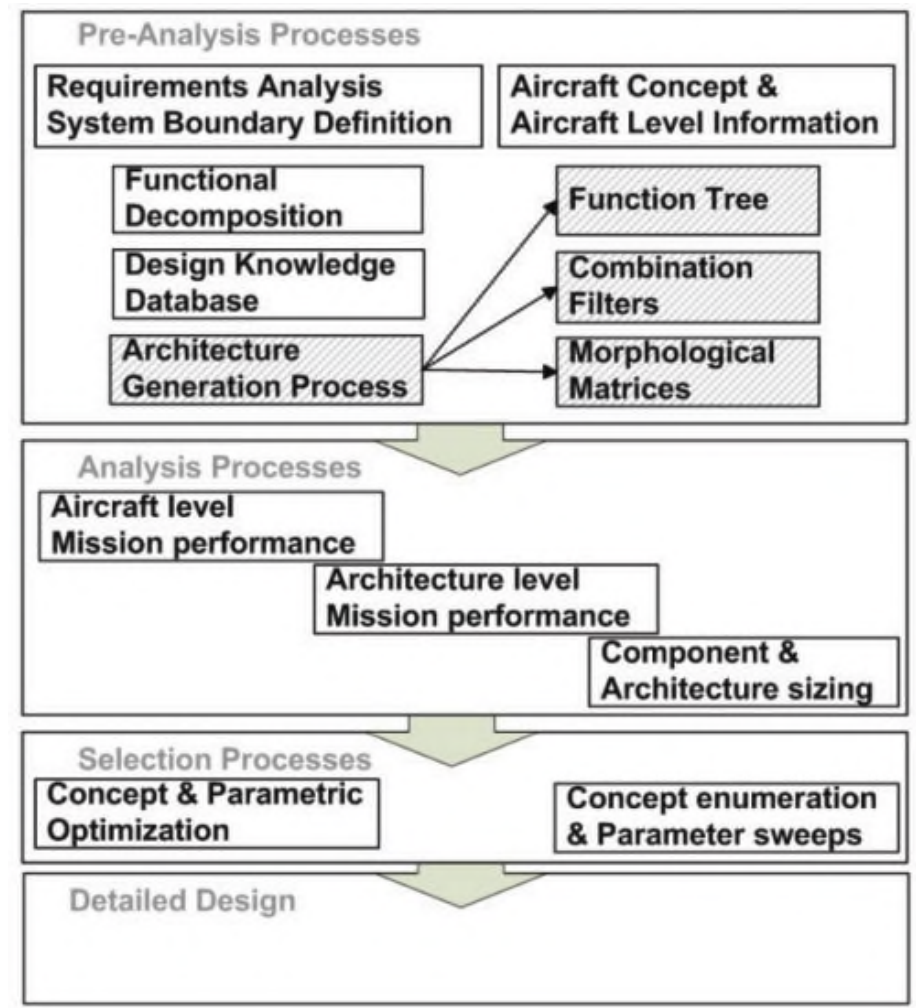

Figure 2: Framework outline 


\section{Hybrid ant colony and genetic algorithm optimisation}

Alternatively to enumeration a global optimisation is possible with a developed hybrid heuristic optimiser, dealing with the highly fractured design space of both architecture composition and parametric design variables. It is composed of a multi-objective ACO routine coupled to a GA. Due to the similarity between the function tree and a travelling sales person type problem (TSP) the ACO approach was chosen. Its effectiveness with such hierarchical problems has been shown to be high. [20] The ACO is applicable to a graph problem as shown in Figure 3 by utilising multiple agents, which generate paths through the function tree and deposit pheromone values on each segment of the total path. The amount of pheromone deposition is a function of the actual competitiveness of a path as evaluated by its objective function. In the case of multiple objectives, the ACO assigns a weighted composite of the objectives to update the pheromone values. The pheromone values function to determine the future probability of choice of the given segment in the tree. In addition, a population of ants is evaluated, kept and only a single competitive member is chosen to update the pheromone values. The ACO method is coupled with a GA exploring the continuous parameter space of the design problem. The process begins by generating a set of random parameter combinations which form the population trait pool. A candidate in the population pool is a path choice in the function tree generated by the ACO. Each of these paths contains certain parameter traits. As an example, consider the path shown in Figure 4 generating an architecture of four components with three continuous component parameters: $\left[\begin{array}{lll}x 12 & x 33 & x 44\end{array}\right]$.
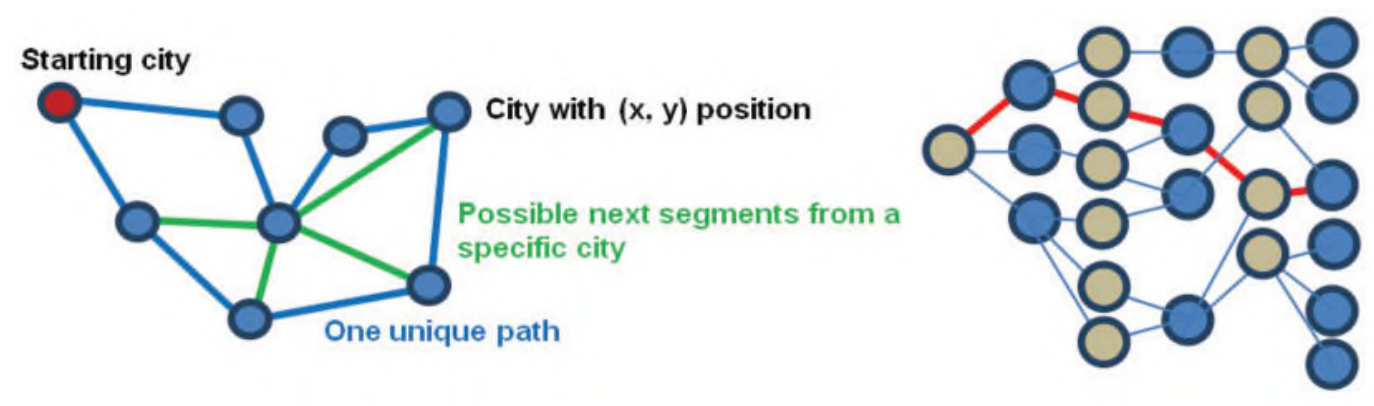

Figure 3: TSP problem and its similarity to the function tree problem

All generated paths are collected in an overall population pool after their parameter assignment. The population is capped at a user-defined limit, after which the initialisation ends and breeding begins. The pool is then utilised to generate the next set of parameters for a newly generated concept path with the ACO procedure. This new set can be represented as the offspring from a breeding process between two or more parents. The chosen parents are always the members from the current population pool, which have the same composition of parameter values and the lowest objective values. If not enough parents of equal composition are available, random agents will take their place. They then start breeding together with a further candidate of randomly generated parameters. This process is shown in Figure 5 for some objective values. The probability to inherit a given parameter trait from the breeding members is given by their respective fitness values and the random agents value calculated with the desired mutation rate. In order to provide a multi-objective optimisation capability, the method incorporates a non-dominant sorting approach and assigns a dummy objective based on the position and distribution of the discovered Pareto front points. This is an a-posterior approach resulting in Pareto fronts or surfaces, which can subsequently be used to select a solution of the most appropriate tradeoffs between objectives, based on its shape. In addition to the front identification, an environment measure of the point spread in the fitness assignment process is included. This is achieved by degrading fitness value of each point by an amount equal to the reciprocal of the distances between it and its nearest neighbours. The connection between the GA and ACO part occurs in multiple places. The given choices of paths in the function tree decides which parameter combinations will be explored. Their values are given by the available past 
paths and new randomly generated ones. If this increases the fitness of the current path and parameter combination, its reproduction is more likely, due to pheromone deposition dependency on the fitness value. The use of a population pool allows for parallelised execution and makes computation of population of concepts more efficient while at the same time allowing for a more diverse exploration. A more detailed optimisation framework description may be found in Judt. [21]

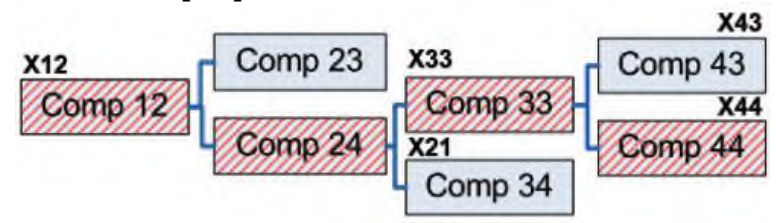

Figure 4: Solution path and its parameter traits

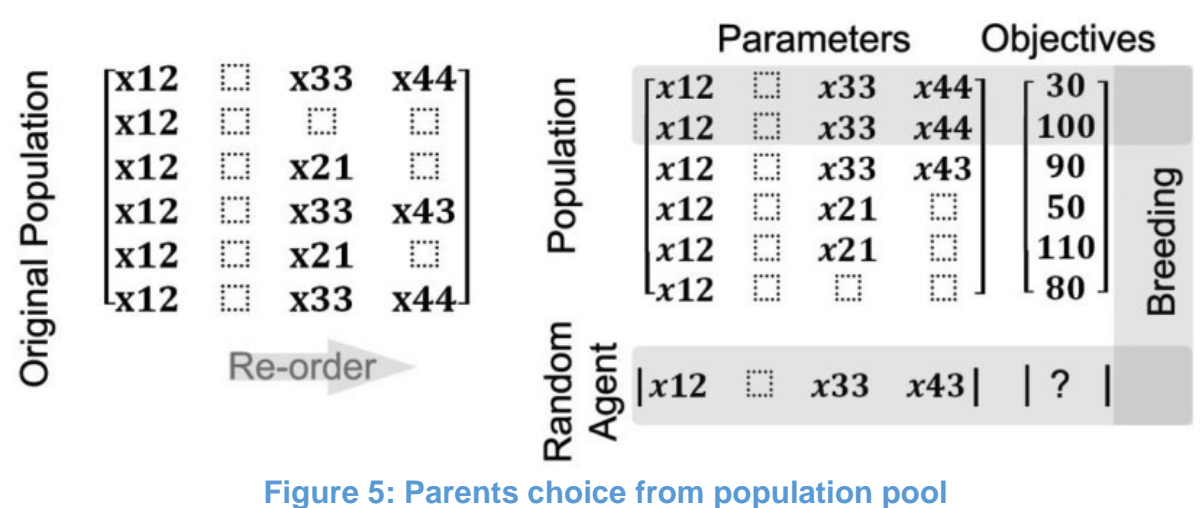

Framework setting in traditional design practice

The described method is merely a part in the overall processes involved in the design of an aircraft as a complex engineering system. Its place within this process, and the required through flows and generation of information will be described with reference to a number of traditional design standards. SAE Aerospace Recommended Practice (ARP) 4754 and 4761 will be discussed as well as the more general ISO/IEC 15288 and the INCOSE Systems Engineering Handbook. [22-25] These documents, together with aircraft systems design textbooks, [26] represent a general overview of the traditional practices employed in today's systems design environments. The ARP guidelines concentrate on establishing the proper processes required for development of a complex aerospace system. A major section focuses on requirement definition, coordination, safety assessment techniques and validation approaches, with a much more detailed description of the various safety assessment techniques provided in ARP4761. Figure 6 gives an overview of the processes, as well as the situation of the proposed method within this structure. Initially the top level aircraft functions are considered through various safety processes methods. The resulting information of failure conditions, effects and classifications is used to assign proper functional requirements to the system level.

These re-iterate through the safety assessment methods, to produce architectural requirements and system architectures, with assigned components. Finally, individual components go through the safety assessment again and implementations of the architectures on a hardware level are considered. Essentially, various iterations of the safety processes are carried out with increasingly detailed input of the design. ISO/IEC 15288 and the INCOSE Handbook summarise the development and life cycle of a system with a number of technical processes, which include the traditional steps such as stakeholder requirements definition and architectural design process. 
Considering these traditional process structures, the described automated method fits in during the architecture definition, analysis and selection phases. By replacing manually defined systems architectures and their analysis models, the potential number of candidate evaluations is amplified and then reduced with the optimiser or enumerative ranking. Other pre and follow-on elements not included in the framework, such as system implementation and integration, need to be considered subsequently with standard approaches.

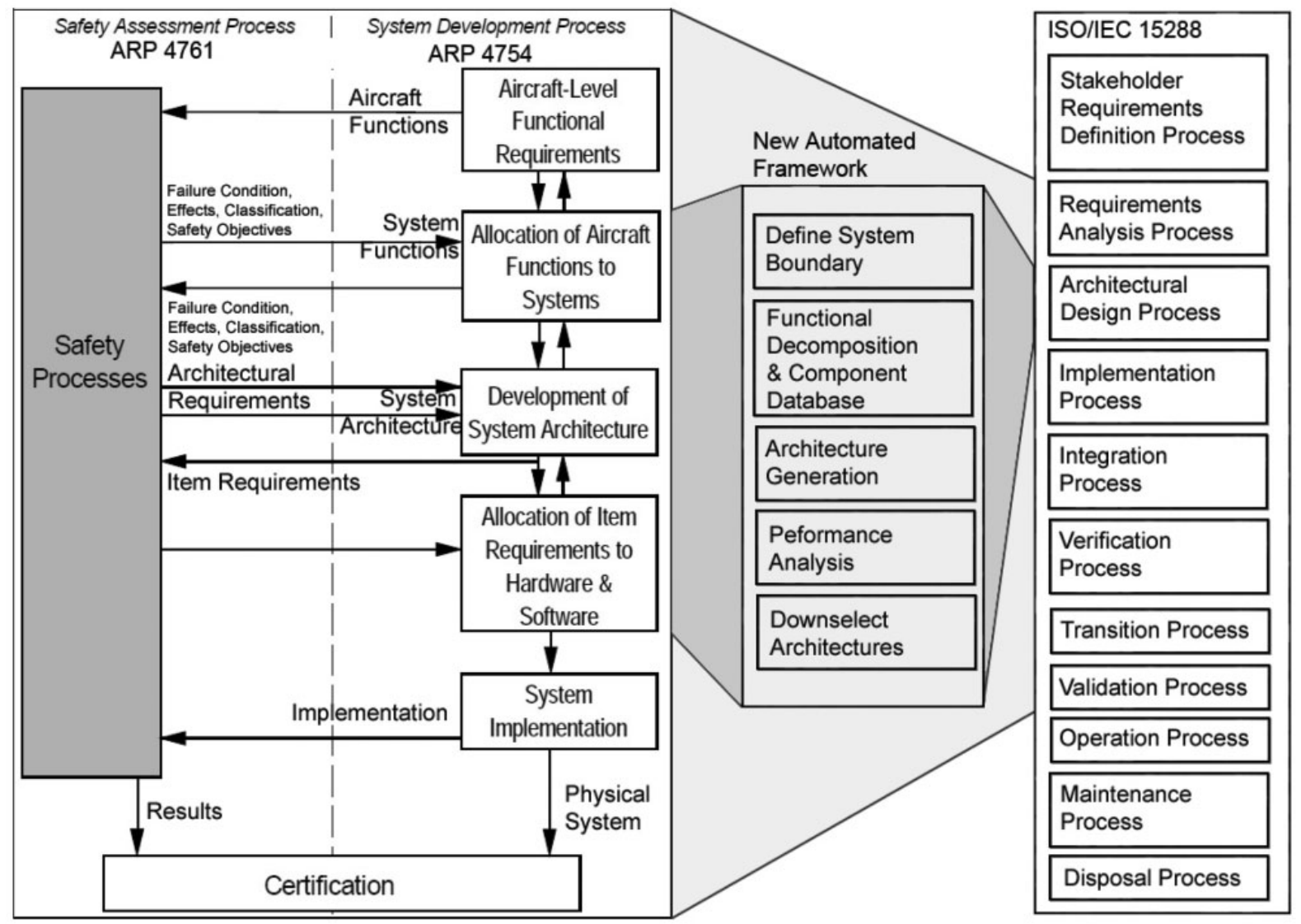

Figure 6: Collection of systems design processes

\section{Framework application}

The framework was developed in conjunction with its application to the design of power and energy systems of a high altitude ultra-long endurance unmanned aerial vehicle (HALE UAV). The main objective of this study focused on the verification of the conceptualised processes and their coherent function in an integrated environment. Even though effort was spent on the development of individual component models from a number of literature sources, [27-34] their verification and setting of parameters was limited. Hence, the produced design data of the UAV subsystems needs to be considered with this in mind. The synthesis of models on the aircraft, architecture and component level, together with concept optimisation for a discontinuous and mixed design space form the main focus of the case study. 


\section{Concept of operations}

The UAV is required to fly loiter and surveillance missions with an endurance of months at altitudes above commercial aircraft operations. These broad requirements for future longterm ground monitoring and communication relay missions, set immediate restrictions on the technology options available for the power system architecture. Consumable fuels as the main energy source are infeasible and a regenerative architecture is required to energy balance the charge and discharge cycle of the storage devices. Technologies such as electric storage systems, fuel cells, electrolysers and photovoltaic (PV) arrays are useful candidates in this context. Hence, the goal is to find the most suitable combination of components which achieves the desired performance while having the best trade-off for the engineering metrics.

\section{Requirements extraction}

With the given problem formulations, the following boundary functional requirements were established:

- Provide high voltage power to the aircraft level (propulsion)

- Provide low voltage power to the aircraft level (payload/external systems power)

- Provide power input (regeneration of energy devices)

By keeping boundary functions general the generation of a regenerative architecture for any component choices during the concept enumeration can be assured. The three functions essentially state that the designed power architecture will require a convertible energy input from the environment, while providing two power outputs to the aircraft level.

In order to provide fault-tolerant architectures, the main electric power distribution channel is dual redundant and a backup energy storage component is included. Such practice is comparable to civil manned aircraft subsystem design practice, where dual to triple redundancy of critical subsystem components is required for certification. [22,35] The backup component replaces the base power provision during a critical failure scenario. It also supplements the base during peak power phases of the mission, such as the take-off and climb segments. This relieves the main power components as they are now sized towards peak power requirements during the cruise and loiter phases only. Similar strategies have been followed by experimental aircraft modification projects, where small general aircraft were refitted with a fuel cell and battery hybrid power system. [36,37] The possibility to include some form of waste heat bottom cycle which recovers part of the energy conversion losses in primary processes to useable power was also investigated. The mission profile shown in Figure 7 includes an initial straight climb out phase followed by turning climb segments until reaching cruise and loiter altitudes. Cruise occurs at best range speed while the turning-loiter is at a radius of $1 \mathrm{~km}$ and best endurance speed. A critical system failure leads to a mission abort scenario where the backup energy storage component is the only power supply, sized to provide enough power for a cruise, descent and land sequence as shown in Figure 8. 


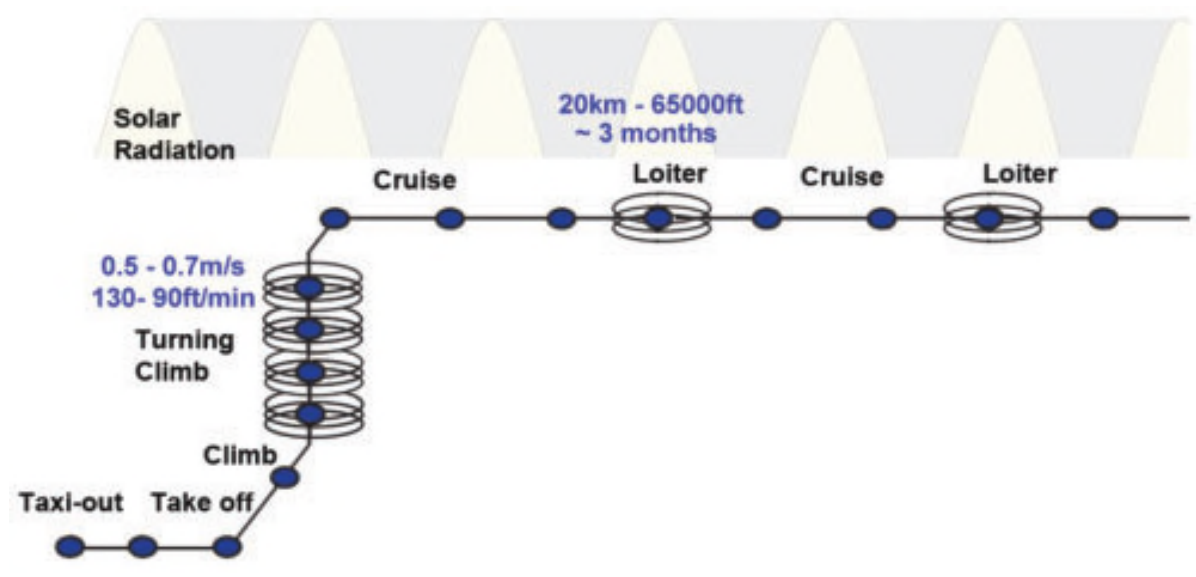

Figure 7: Standard mission profile

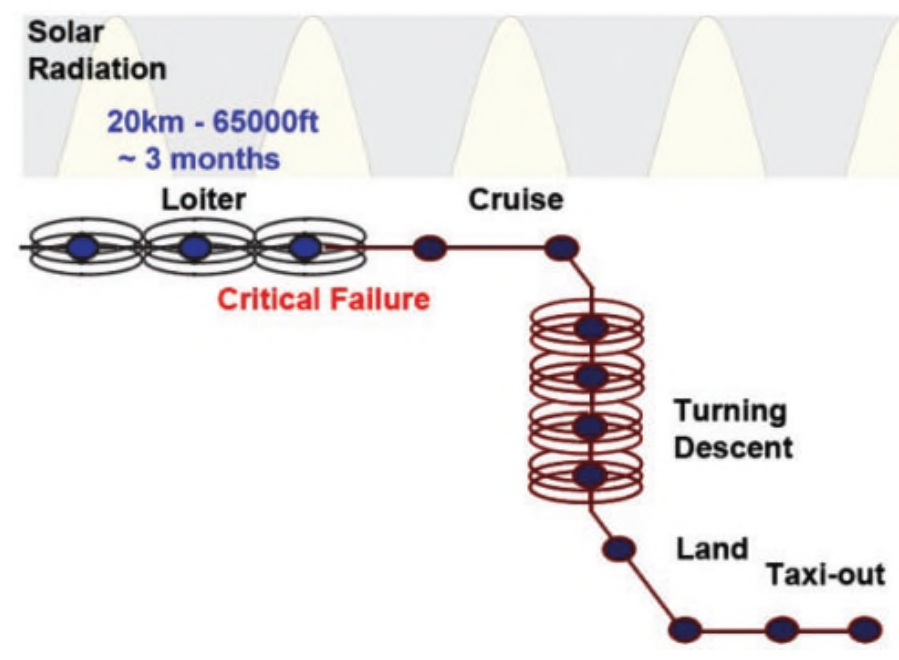

Figure 8: Failure mission profile

\section{Aircraft and external models}

Predicting the aircraft subsystem performance requires models of aircraft mission performance and external influencing factors such as the atmosphere and solar radiation power. Hence, the aircraft concept itself needs to be available in terms of its geometric, aerodynamic and mass data. The size of the aircraft concept is partly determined by the aircraft performance calculations and partly fixed. Fuselage dimensions are taken as fixed, as well as the wing loading and the wing aspect ratio. As new maximum take-off weight (MTOW) values are produced by the aircraft performance analysis, a resizing of the wing reference area takes place, leading to a new span value for the wing. Hence, the method captures the snowball effect resulting from changes in aircraft mass and subsequent structural changes. Figure 9 gives an overview of the standard concept geometry.

For this case study, a conservative configuration was chosen consisting of a high aspect ratio wing and conventional tail. Aspects of the geometry, such as wing airfoils, taper ratio and sweep, are assumed as fixed during the methodology, since the focus is on architectural systems analysis rather than top level aircraft design optimisation. The aircraft platform geometric information is used to estimate aerodynamic parameters and aircraft masses, with the mission performance calculations then estimating propulsive power requirements. Traditional aerodynamic conceptual design equations where used to establish lift and drag coefficient data in dependence of aircraft geometry, and flight condition.[1,2] 


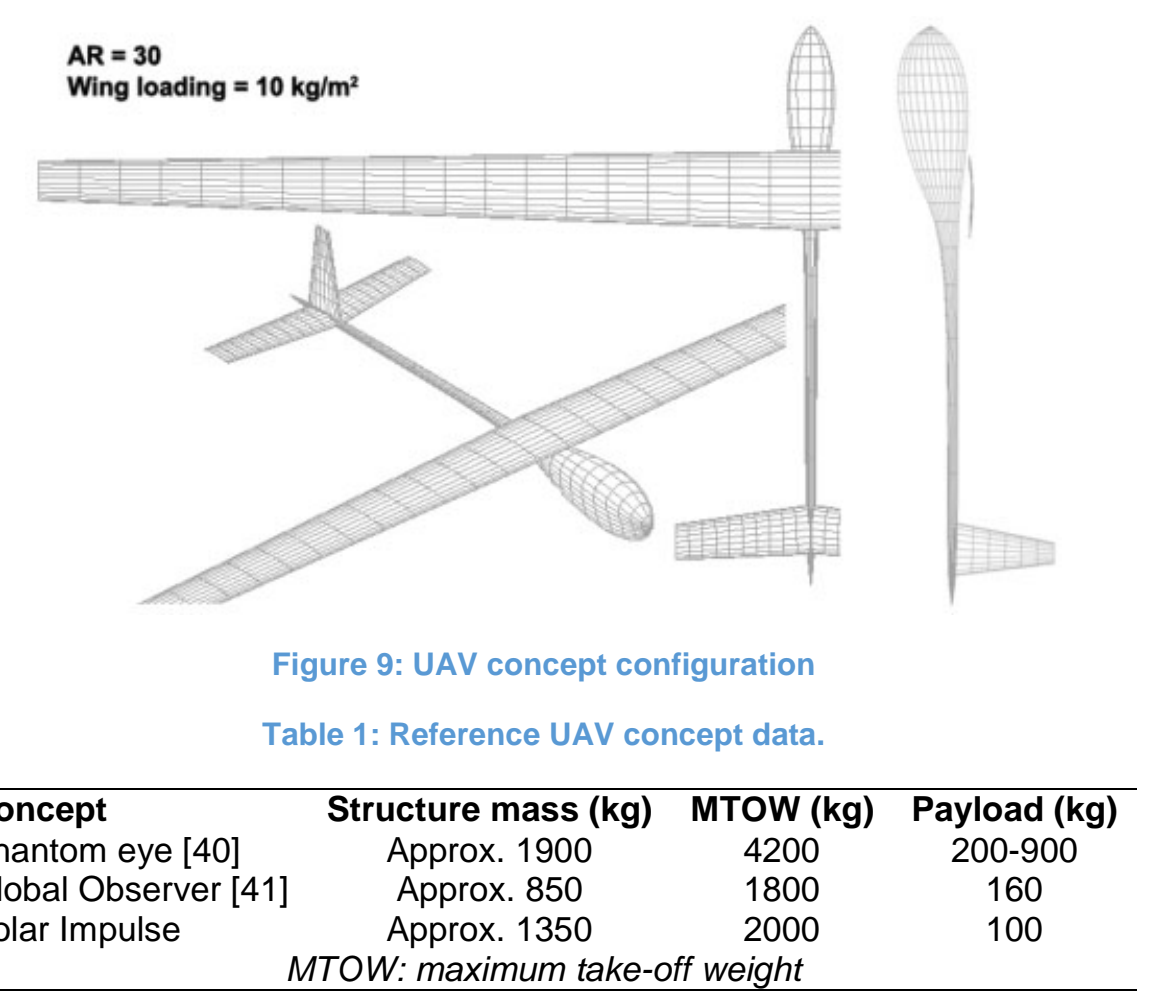

Since the UAV will likely require advanced structural designs and materials, the estimation of the empty mass should not be made with traditional aircraft design equations. Instead the case study bases aircraft structural mass on similar vehicles, such as the 'Solar Impulse HB-SIA. $[38,39]$ These designs are high span, high aspect ratio vehicles with predominantly carbon composite construction. The solar impulse itself is not an unmanned vehicle, and so has to provide a number of human accommodating measures, such as increased fuselage space and comfort, for a single pilot. With a take-off mass of $2000 \mathrm{~kg}$ a battery mass of 450 $\mathrm{kg}$ and a payload of around $100 \mathrm{~kg}$, its structural empty mass is estimated to be around 1350 $\mathrm{kg}$. A linear relationship of empty mass increase with MTOW is assumed and established with data for a few further concepts as shown in Table 1 and equation (1).

Structural mass estimation of an advanced UAV:

$$
M_{\text {struc }}=475+0.32 * M_{\text {MTOW }}
$$

Equation 1

Aircraft mass breakdown:

$$
M_{\text {MTOW }}=M_{\text {struc }}+M_{\text {Arch }}+M_{\text {pay }}
$$

Equation 2

Some of the potential architectures have a constant mass throughout the mission, whereas others change in mass during the charge and discharge cycle of the main energy storage devices. This requires a flexible approach to the aircraft performance modelling, to be able to analyse both of these conditions. Rather than using the well-known Breguet-range approach where fuel burn equals aircraft mass change during steady level flight, the problem is approached with a numerical solver. Equations (3) and (4) present the two different approaches, with the numerical one considering the amount of energy required $E_{\text {storage }}$ to cover the range $R_{\text {seg }}$ of each segment at starting architecture mass $W_{\operatorname{Arch~}, O}$.

Breguet-range equation for steady cruise with fuel burn:

$$
-\frac{d W}{d t}=g * m_{f} \stackrel{\text { yields }}{\longrightarrow} R=\frac{V}{c_{t}} * \frac{L}{D} * \ln \frac{W_{\text {seg start }}}{W_{\text {seg end }}}
$$


Numerical approach with variable aircraft mass change:

$$
-\frac{d W}{d t}=g * m_{f} \stackrel{\text { yields }}{\longrightarrow} R_{\text {seg }}=F\left(V, \frac{L}{D}, E_{\text {storage }}, W_{\text {Arch }, o}\right)
$$

The architecture mass-time profiles are used as inputs to equation (2), rather than a single aircraft take-off mass value. Therefore as the aircraft performance is estimated, mass changes during each time step are available from the past estimate of the architecture performance. These profiles originate from the architecture level analysis, where mass and power flows between components and the aircraft are computed. New aircraft level results then update these mass profiles and the process is repeated. An outline of the aircraft level modelling is provided in Figure 10 with $M_{\text {Arch }}$ computed per mission time step from architecture level analysis. Apart from models of the standard atmosphere, a further model of the sun's radiation intensity was required. Here various inputs need to be taken into account, including the time of day, day of year and position in terms of latitude and altitude. These models are well established and available. [42, 43]

Architecture concept space: One of the advantages of the enumerative method is the fact that a much larger space of concepts can be generated and analysed without much work associated with setting up individual architectural model tools. To reinforce this deduction, various types of components were included in the case study, from pure electric systems with battery storage, to fuel cell powered and heat recycling bottoming cycles. Figure 11 depicts the function tree of architectures.

Fuel cell architectures will require multiple components for storage, transport and maintenance of reactants, including heat exchangers and tanks. The general principles of the fuel cell type architectures are the same for the various cell types. The regenerative system is established, by converting daytime solar radiation to electric current, which splits water into hydrogen and oxygen. The hydrogen is then stored in a tank, for reuse in the fuel cell, during night operations. Oxygen may be stored on board, producing a fully closed mass loop system, or extracted from the atmosphere, when required with an electric compressor. A secondary battery functions as a backup system, when peak and emergency power are required. This could be replaced by a further fuel cell system, or emergency combustion engine. As is evident, design options are abundant and for this case study just a snapshot is chosen, generating an architecture space of 146 unique candidates.

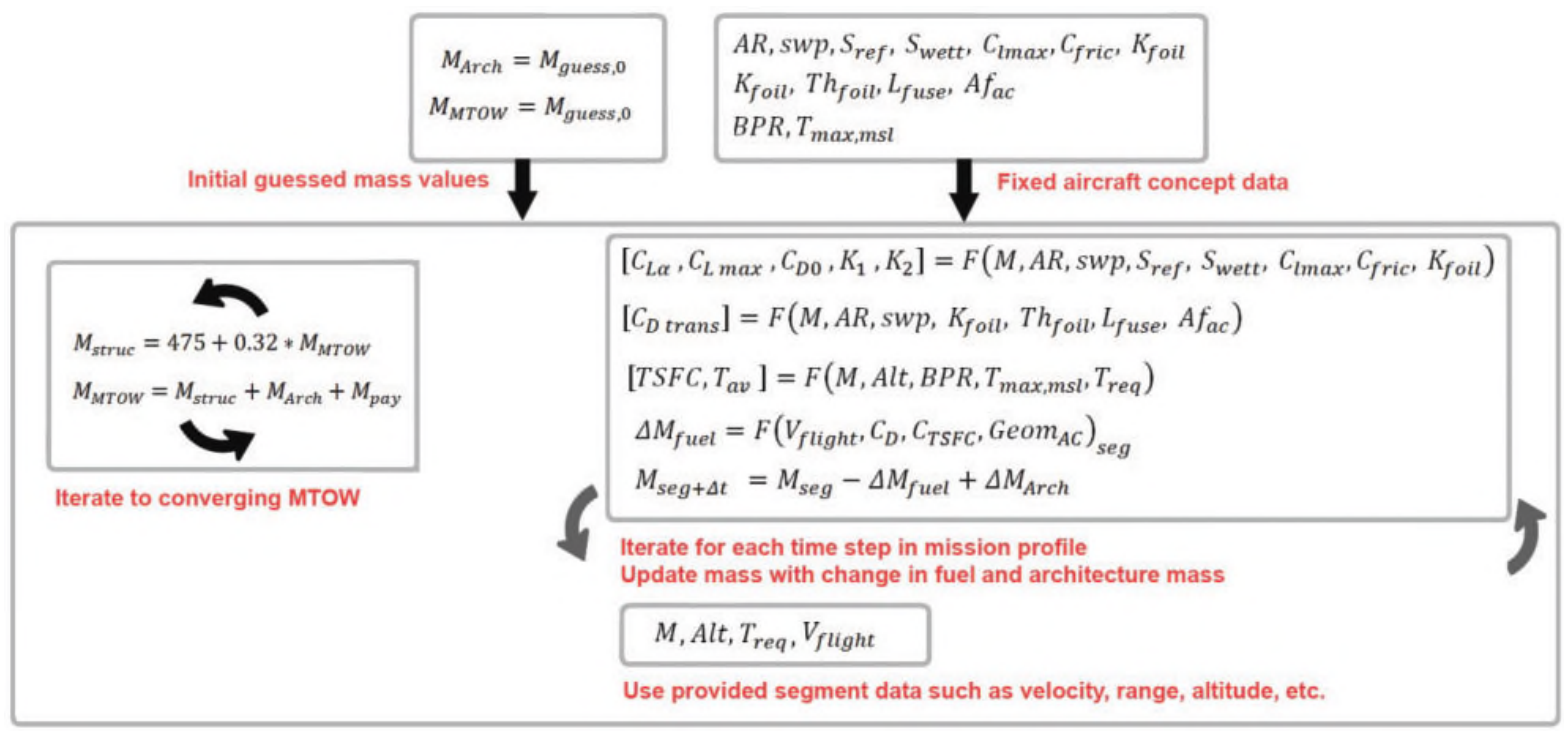

Figure 10: Iterative approach for mission performance of a variable aircraft and subsystem mass 


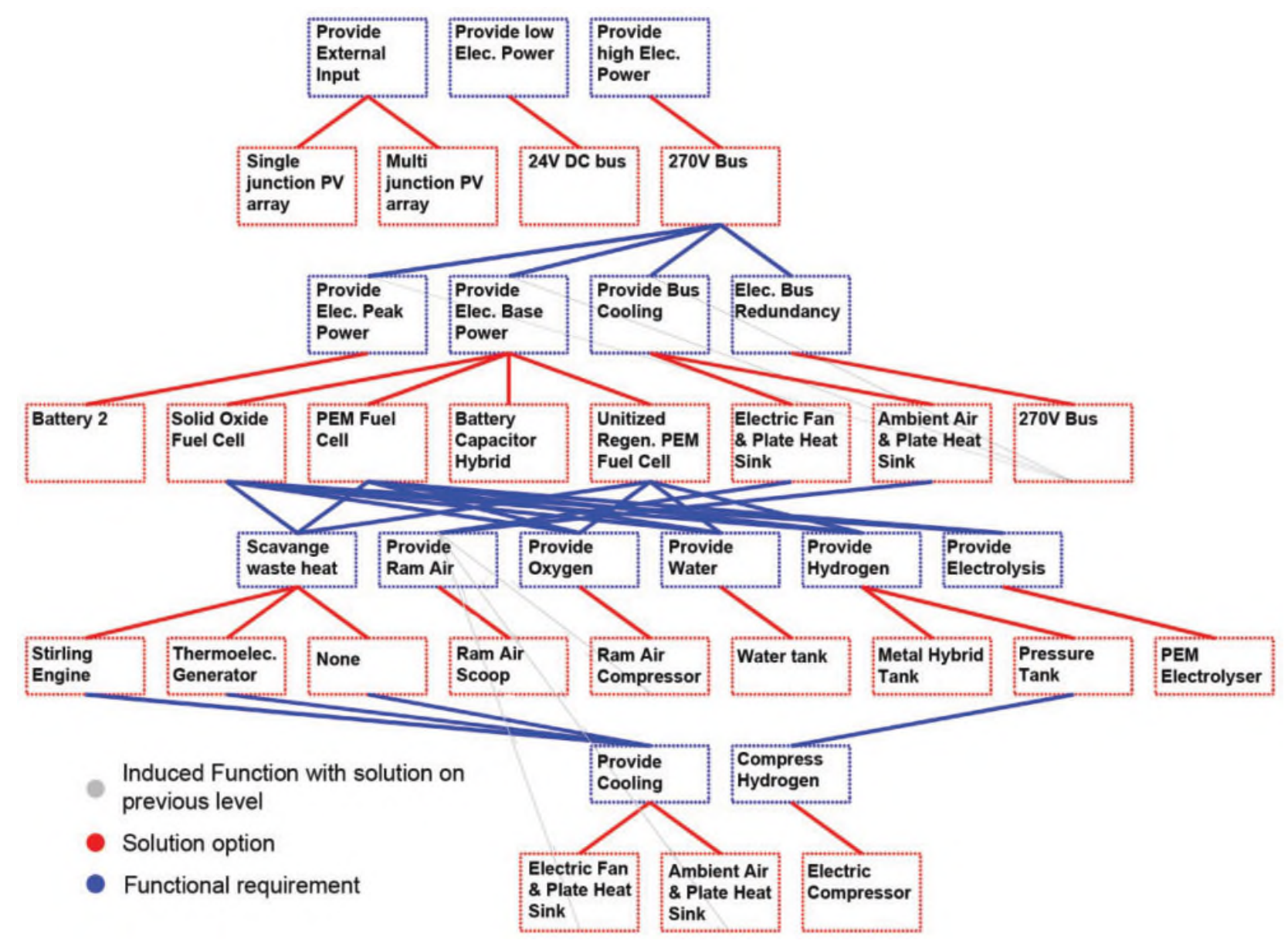

Figure 11: Case study function tree.

PV: photovoltaic. PEM: Proton exchange membrane

\section{Component models}

Each physical solution to the functional requirements of the subsystem is represented by a mathematical model of its performance and engineering metrics. Hence, fuel cells are modelled in terms of power output, reactant flow rates and heat generation, to transmit the required input and output values to storage elements, and heat exchangers.

The static design parameters of the various models, were populated with initial values and then either adapted through the sizing mechanism, or left as a variable for optimisation studies of the design problem. The engineering metrics of importance in this study were architectural mass, power ratio and complexity. Values were estimated with links to model and architecture parameters as shown in Table 2. For example in the case of a battery electric storage system, its mass $M_{i}$ may be estimated by the required energy and power deliveries. A heat exchanger's mass is usually linked to its dimension and surface area, a fuel cell to the number of cell stacks and active reaction area and an electric bus to the maximum electric current flow through it, as well as the cable length of connections. The architecture power ratio compares the peak output power and heat dissipation of the architectures, hence indicating how efficiently the outputs are provided in terms of heat dissipation requirements. The complexity of architectures was modelled by assigning a component specific value, increasing with the number of components connections and types. Hence, architectures with more components and connections with many different types of exchanges are more complex. Each architecture has $N$ components, $N_{\text {con }}$ connections, $C_{c o n}$ constants for each component and Con $_{\text {type }}$ constants for each connection 
type. These additional constants serve to describe the complexity impact due to the use of different forms of power transfer such as electric, hydraulic or fuel flow and are either set as fixed by the authors, or defined as a function of other component metrics. Hence for most components this results in a direct relationship between component mass and complexity. The maximum heat dissipation of an architecture is given as $H_{A r c h}$ at the current maximum power output level $P_{\text {Arch }}$ during the flown mission.

Table 2: Architecture design metrics

\begin{tabular}{|c|c|c|c|}
\hline Metrics & Objective & Function & Constants \\
\hline Mass & Minimise & $M_{\text {Arch }}=\sum_{i=1}^{N} M_{i}$ & - \\
\hline Power ratio & Maximise & $P R_{\text {Arch }}=\frac{\max \left(P_{\text {Arch }}\right)}{H_{\text {Arch }}}$ & - \\
\hline Complexity & Minimise & $C_{\text {Arch }}=\sum_{i=1}^{N}\left[C_{i} *\left(\sum_{j=1}^{N_{\text {con }}} C_{\text {con } j} *\right.\right.$ Con $_{\text {type } j}$ & $\begin{array}{l}C_{i}=F\left(M_{i}, P_{\text {Arch }}, i\right) \\
C_{\text {con } j}=\text { Fixed } \\
\text { Con }_{\text {type } j}=\text { Fixed }\end{array}$ \\
\hline
\end{tabular}

\section{Standard scenario results}

The standard mission profile, as outlined in Figure 7, consists of a take-off at 17:00 hours, climb out at an initial $130 \mathrm{ft} / \mathrm{min}$ dropping to $90 \mathrm{ft} / \mathrm{min}$ at $4000 \mathrm{ft}$ until reaching 60,000 ft cruise and loiter altitude. The payload mass is $200 \mathrm{~kg}$ with a fixed power consumption of $2 \mathrm{~kW}$. The take-off time is chosen to correspond to a peak power demand during the end of climb phase occurring just before sunrise, making this a critical case. The latitude and day of the year are fixed at 55 north and 8 June, corresponding to an example vehicle deployment in the United Kingdom during favourable summer conditions. The plots in Figure 12 show results from aircraft and external models of a candidate architecture design. Peak power demands are around $35 \mathrm{~kW}$ during the last climb segment of $90 \mathrm{ft} / \mathrm{s}$ before reaching cruise altitude. Cruise and loiter power consumption is oscillating around the $18 \mathrm{~kW}$ mark, as the mass of the architecture changes. Results are produced for two full day and night cycles only with fixed latitude. In order to guarantee desired mission performance during the whole 30 day endurance period, the critical 2 days of the year and latitude for such a mission needs to be chosen for this analysis. This analysis and sizing to such a critical case assures energy balanced operation during the whole mission.
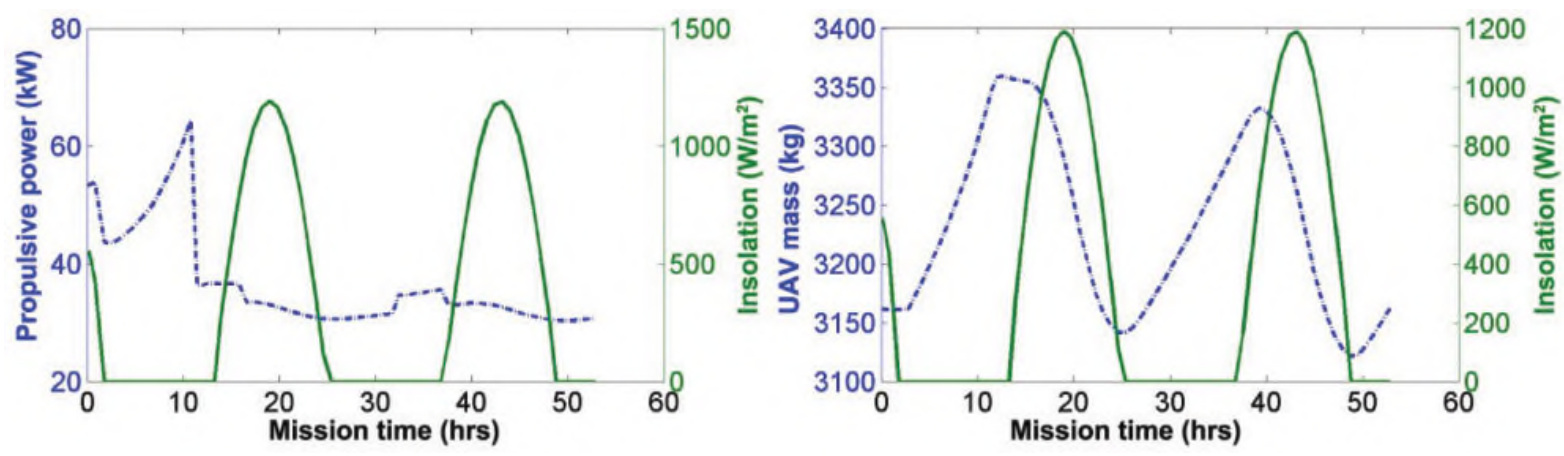

Figure 12: Aircraft level performance for a single UAV architecture 
The aircraft and architecture mass breakdown is presented in Figure 13, with a take-off mass of $3160 \mathrm{~kg}$ and a structural mass fraction of 0.47 . The second major part is the power subsystem at a mean mass of $1580 \mathrm{~kg}$ and a variation of up to $100 \mathrm{~kg}$, due to the gain in oxygen from the atmosphere during the charge and discharge cycles. The tanks for hydrogen and water take up a major part of the mass, together with the backup battery. This is explained by the low specific energy values of the battery pack for the given energy requirements and high structural mass requirements for low the hydrogen to be stored.

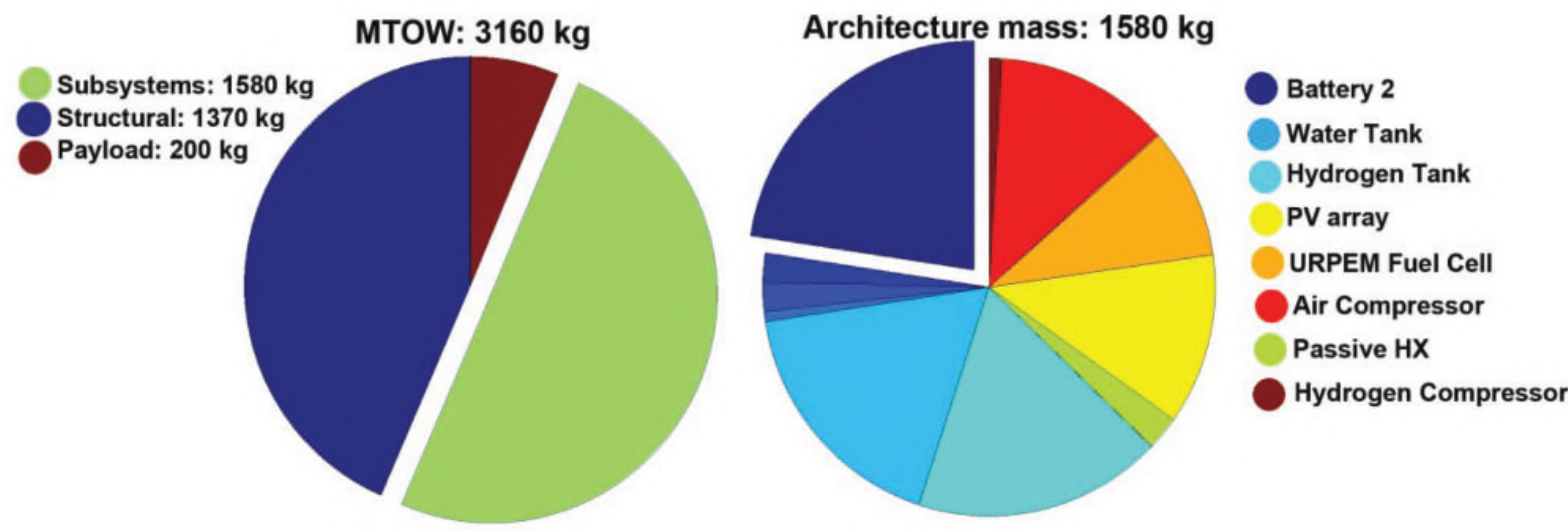

Figure 13: Mass-breakdown of a subsystem architecture and aircraft URPEM: unitized regenerative proton exchange membrane.

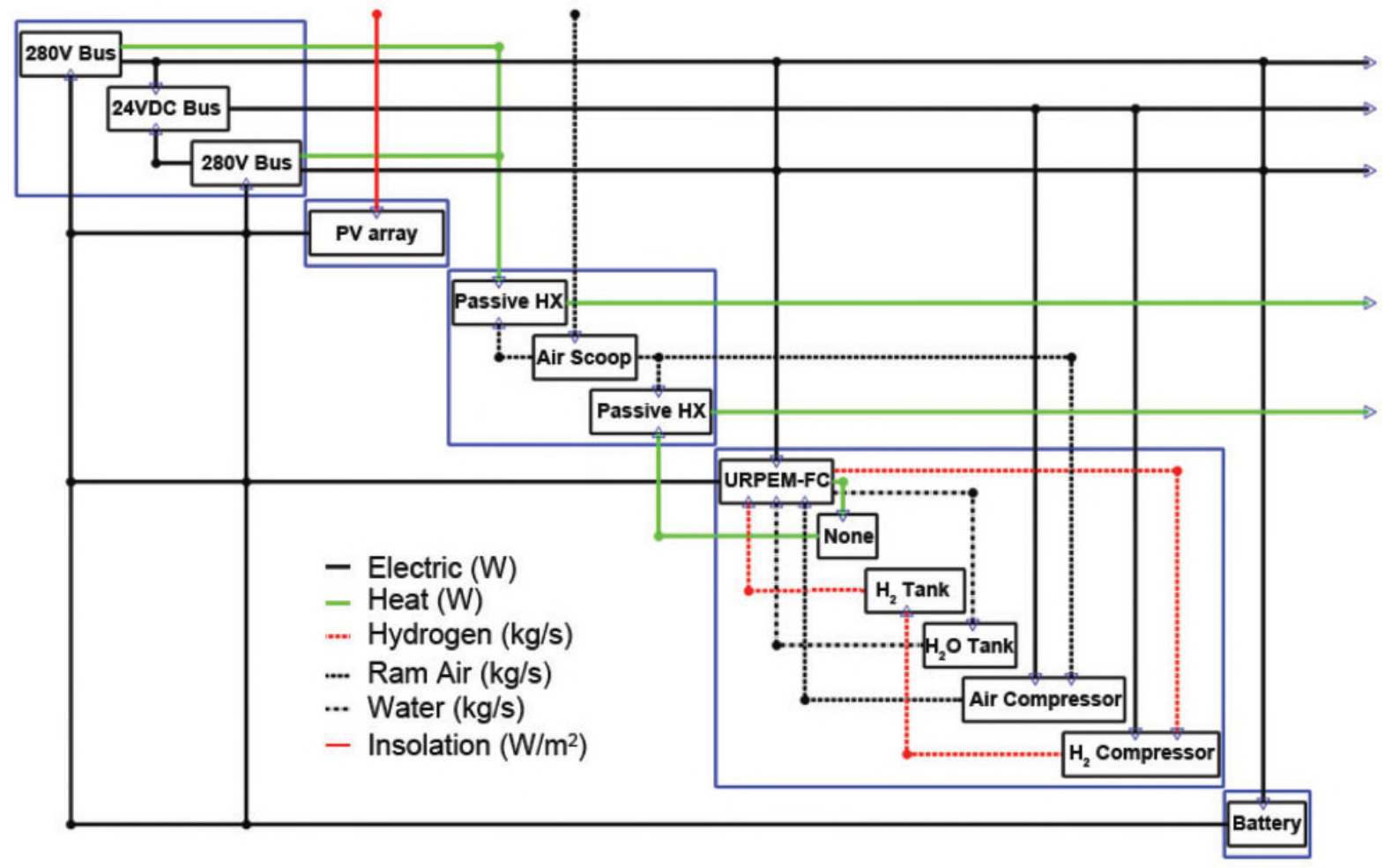

Figure 14: Example concept architecture component structure. FC: Fuel Cell

The component interaction structure of the example architecture is shown in Figure 14. Each component performance model exchanges input and output variable types with other subsystem elements. Additionally components have states, such as temperature and state of charge of energy storage devices, which are driven by the performance models and serve as inputs to other models, as well as performance objectives in the component sizing routine. Results for the complete set of enumerated and sized architectures are presented in 
Figures 15 to 18. Each dot in the scatter plots represents a fully sized and converged architecture. Figure 15 categorises concepts according to technology used for the main power provision, and shows sliced plots of architecture mass, power ratio and complexity design metrics. In terms of power ratio and architectural mass, solid oxide fuel cell (SO-FC) concepts outperform most alternatives, with a combination of low mass and high power ratio. The complexity metric is mainly driven by the number of architecture components, which is higher for the SO-FC concepts and the lowest for the unitised regenerative proton exchange membrane fuel cells (URPEM-FC). Hence, a clear discontinuity is visible in Figure 15. For the two hydrogen tank options, the pressure tank tends to produce lighter system concepts for the current study and assumed model parameters. Even though a hydrogen compressor is required the concepts still outperform the much heavier metal hybrid tank concepts in terms of total architecture mass. This is shown in Figure 16. No architectures with pure battery systems for energy storage and power provision resulted in feasible solutions after sizing. This is attributed to the fact that such components modelled with today's technology parameters have low specific energy. Investigations into their competitiveness with future higher values were carried out and are presented in a later section.
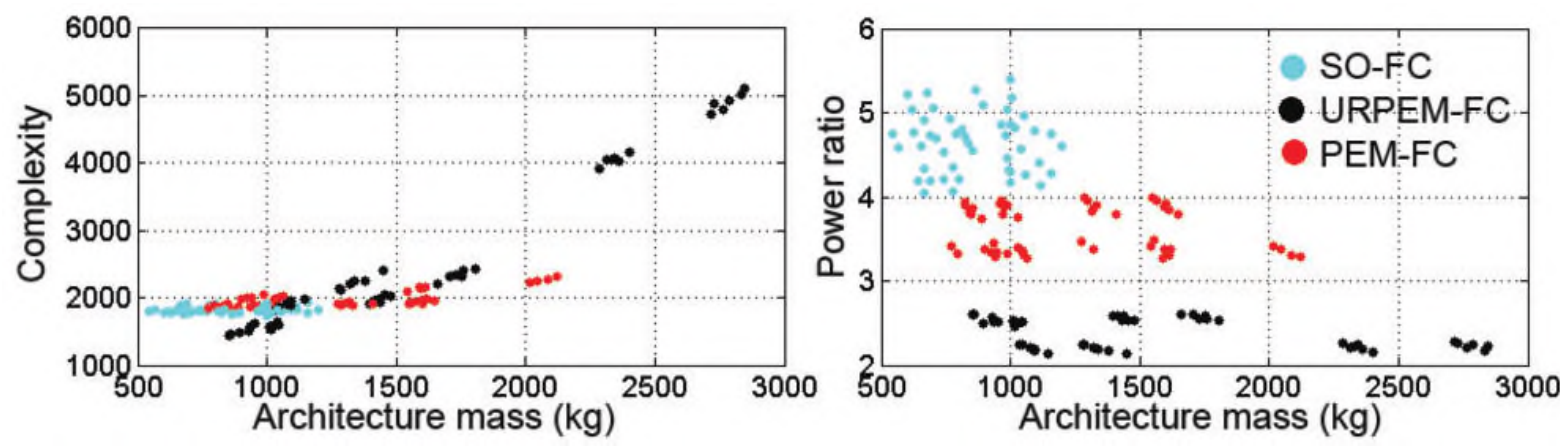

Figure 15: Architecture design metric slices with base power categorisation
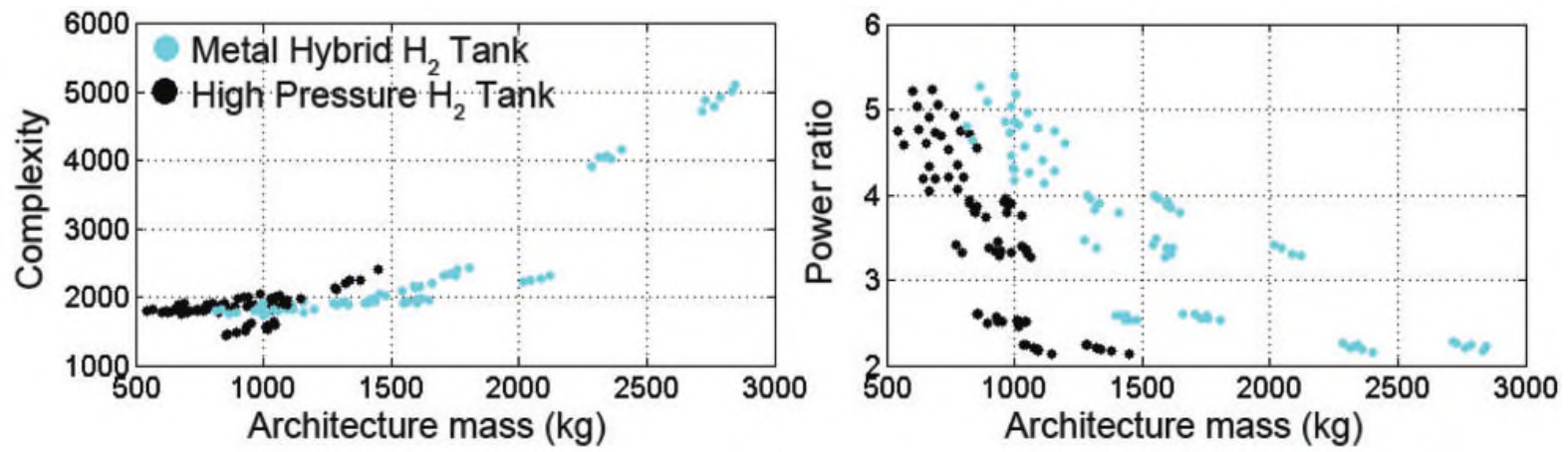

Figure 16: Architecture design metric slices with hydrogen storage categorisation

Another categorisation of the architectures is presented in Figure 17, focusing on the possible solutions for the waste heat recovery system. A thermoelectric generator may be used to convert a waste heat flux into a voltage potential difference and current flow.

The other option, a Stirling engine, employs a cyclical process of fluid expansions and compression to convert the heat flux into a shaft rotation, which drives an alternator for generation of electric power. Both processes require additional components, a large enough temperature difference and a heat sink to dump low grade heat with the help of passive or active heat exchangers. 
In this case study, the enumeration results show only a minor difference in design metrics for using such systems in terms of system mass. In combination with the SO-FC a larger impact is observable in Figure 17, where SO-FC concepts which include TEGs achieving a higher power ratio and a slightly lower architecture mass, compared to same concepts without a TEG. Generally speaking though waste heat component influences are too small compared to other component choice effects, due to their low conversion efficiency. Figure 18 shows another categorisation according to the choice of PV arrays. Single and multi-cell PV arrays are modelled as having different nominal cell efficiency and the cell mass per area. This results in a clear segmentation in the architecture mass direction with such components. A slightly heavier PV array with higher efficiency outperforms the alternative option in all concept compositions.
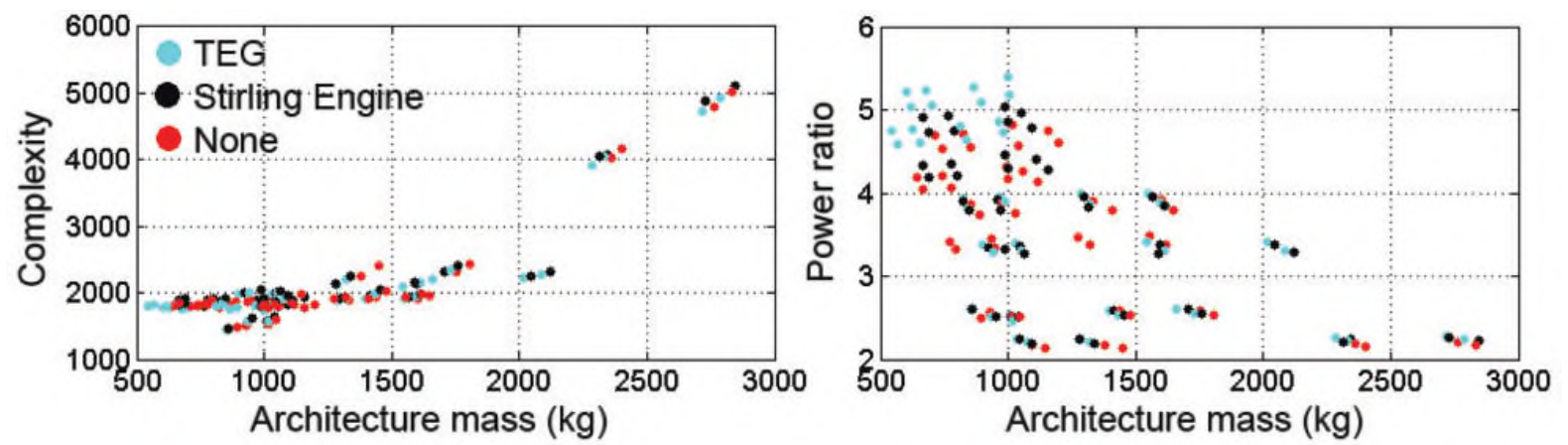

Figure 17: Architecture design metric slices with heat recovery categorisation
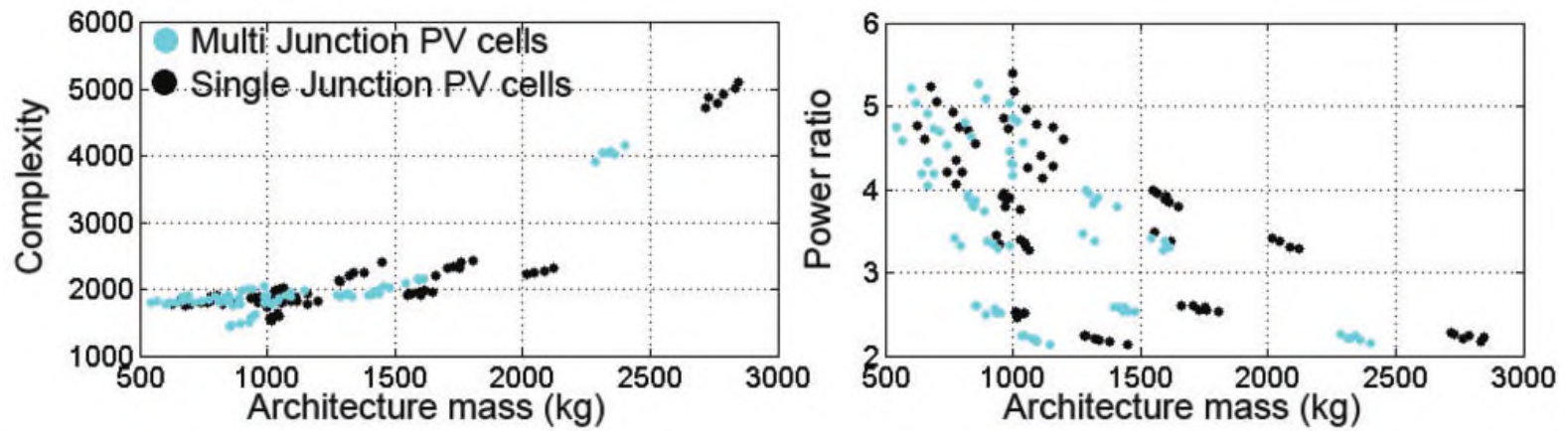

Figure 18: Architecture design metric slices with photovoltaic (PV) array type categorisation

\section{Parameter sweep results}

After producing a set of feasible and competitive architectures, further analysis of their variability to design parameters is appropriate. A number of different changes were investigated and categorised as shown in Table 3.

Rather than evaluating the whole architecture concept space, just three competitive architectures were chosen. Two are based on a SO-FC with either a waste heat recovery system or direct heat dissipation, and the third is a battery only concept. The rationale behind this choice stems from the fact that SO-FC concepts outperformed other concepts for the design metrics, as was shown in the slice plots in Figures 15 to 18. The pure battery concept investigates the technology parameters required to become a feasible design. Figures 19 and 20 show a linear change in architecture mass due to increased payload for both architectures, where as a change in PV array cell efficiency provides a decaying improvement in architecture mass reduction. For changes in battery specific energy, the battery only architecture starts to converge around the $600 \mathrm{Wh} / \mathrm{kg}$ mark as is observable in Figure 21. Due to the low component count and waste heat generation of such a pure 
electric architecture, its complexity and power ratio metric immediately outperforms both other candidates. In addition, there is also a mass reduction taking place for the SO-FC concepts, as the backup battery is resized accordingly

Table 3: Parameter sweep range

\begin{tabular}{lll}
\hline Parameter & Range & Type \\
Payload mass & $50-350 \mathrm{~kg}$ & Aircraft \\
PV array nominal efficiency & $20-60 \%$ & Component \\
Battery specific energy & $300-1200 \mathrm{Wh} / \mathrm{kg}$ & Component \\
Cruise/ Loiter altitude & $12-22 \mathrm{~km}$ & Mission \\
\hline
\end{tabular}

For variations in the cruise and loiter altitude, a steep divergence in architecture mass is observable around the $22 \mathrm{~km}$ mark, as shown in Figure 22. This is partly due to the large increase in backup battery mass, to satisfy the higher propulsive power requirements for the mission abort cruise segment. In addition, the hydrogen system components also increase in mass and size, due to the resizing to meet the higher power demands at such altitudes.
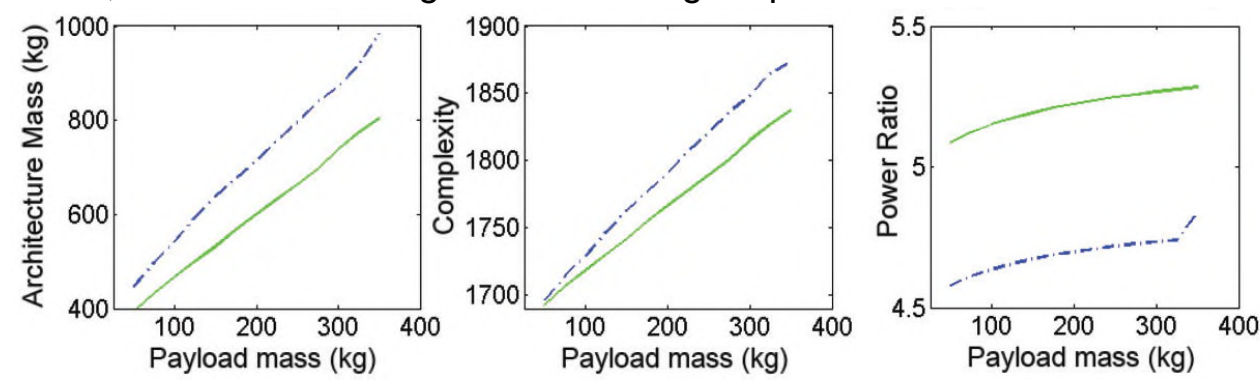

Figure 19: Payload mass parameter sweep
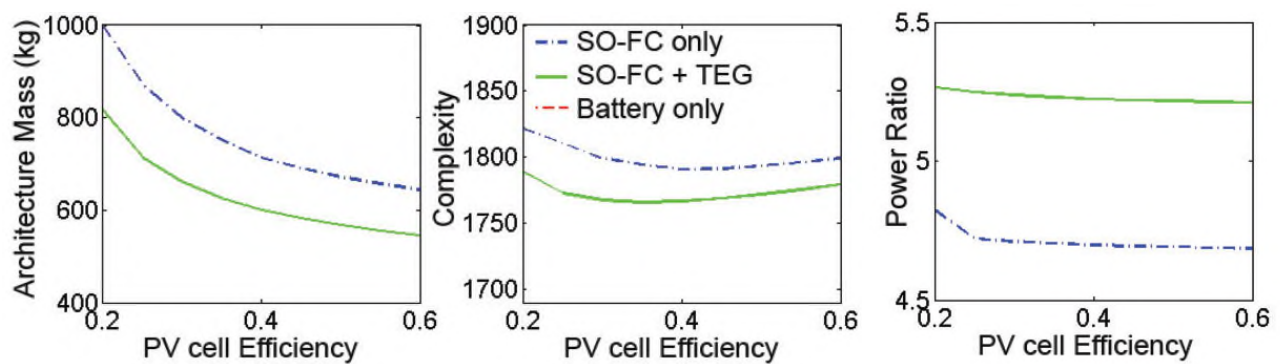

Figure 20: PV cell efficiency parameter sweep
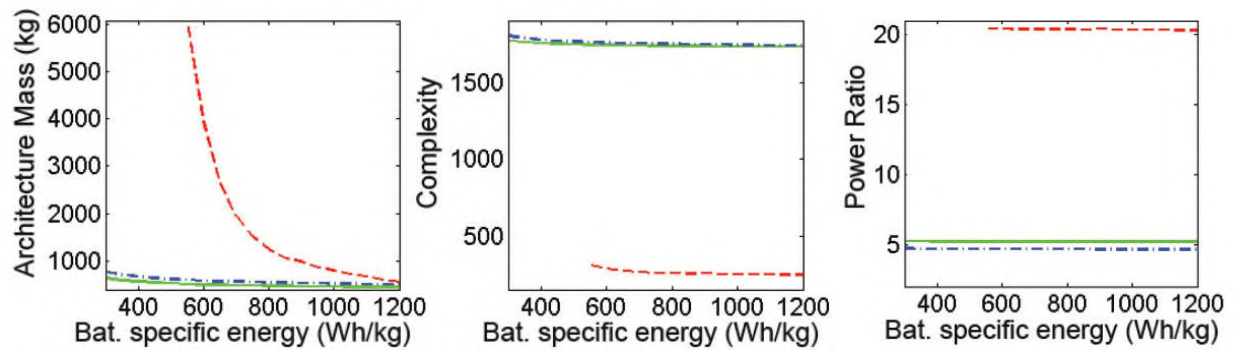

Figure 21: Battery specific energy parameter sweep
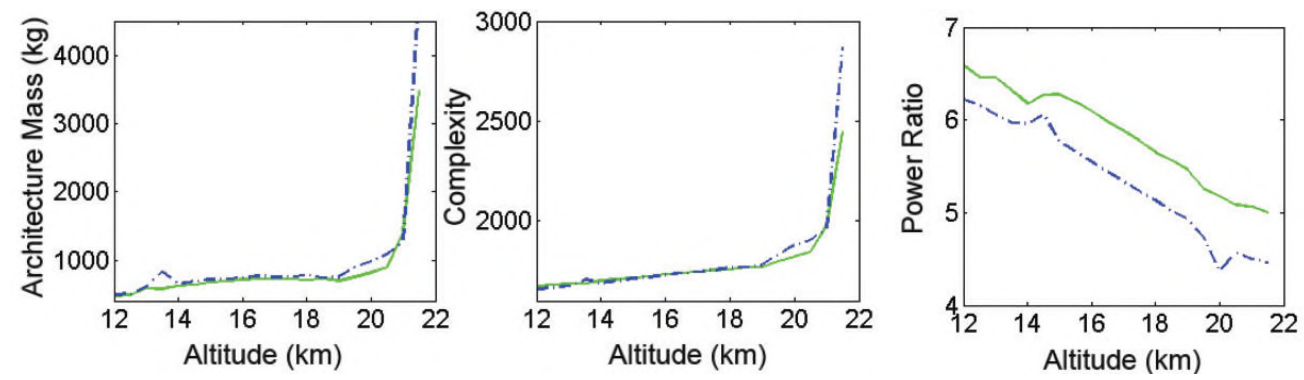

Figure 22: Cruise/ Loiter altitude sweep 


\section{Optimisation results}

As an alternative to concept enumeration and parameter sweeps, the optimisation over both the concept and parametric space is undertaken with the hybrid optimiser. The optimisation problem can be stated in a general from as in equation (5). Results will be presented for three distinct cases, as outlined in Table 4. $P_{\text {mission }}$ refers to the parameters of the mission segments to build the flight profile, $P_{\text {Aircraft }}$ to the aircraft concept parameters and $P_{\text {Architecture }}$ to the architecture component parameters, which are given by the $F T_{\text {choices }}$ choices made in the function tree and the $P_{\text {Arch,database }}$ component parameters in the database.

General optimisation problem:

Equation 5

$$
\begin{gathered}
\min F\left(P_{\text {mission }}, P_{\text {Aircraft }}, P_{\text {Architecture }}\right) \\
P_{\text {Architecture }}=F\left(F T_{\text {choices }}, P_{\text {Arch, database }}\right)
\end{gathered}
$$

Single objective optimisation for a fixed parameter, varying concept space and the converse are shown in Figures 23 and 24. Study A1 deals with a fixed parametric space where only the ACO algorithm is active as different paths in the function tree are explored. The convergence of this process is determined by the changes in the pheromone values shown in Figure 23 for a number of path segments of the function tree. Competitive architectures continuously increase their path segment values until the evaporation and deposition is equal. Repeatability of the heuristic optimiser is tested with multiple runs of equal initial conditions. Repeated convergence consistently leads to the segments which belong to the generation of a concept containing a SO-FC; a high pressure hydrogen tank and a TEG maintain the highest pheromone levels as paths are evaluated. Hence in terms of pure mass minimisation for fixed parameters, the optimiser will discover the same concept as was produced during the enumeration shown in Figures 15 to 18.

Table 4: Optimisation cases

\begin{tabular}{ccccc}
\hline Study Objectives & Parameters & Parameters & Range & Identifier \\
& $P_{\text {mission }} P_{\text {Aircraft }} P_{\text {Arch,database }}$ & $F T_{\text {choices }}$ & & \\
Minimise MTOW & Fixed & Variable & - & A1 \\
Minimise MTOW & Payload mass & Fixed to one & $50-350 \mathrm{~kg}$ & $\mathrm{~A} 2$ \\
& PV array efficiency & choice & $20-60 \%$ & \\
& Cruise/ Loiter altitude & & $12-20 \mathrm{~km}$ & \\
Minimise MTOW & Payload mass & Variable & $50-350 \mathrm{~kg}$ & $\mathrm{~B} 1$ \\
$\&$ & $\mathrm{PV}$ array efficiency & Variable & $20-60 \%$ & \\
Minimise Complexity & Cruise/ Loiter altitude & & $12-20 \mathrm{~km}$ & \\
& $\mathrm{H}_{2}$ tank pressure & & $300-700 \mathrm{bar}$ & \\
& Battery specific energy & & $300-1200$ & \\
& & $\mathrm{Wh} / \mathrm{kg}$ &
\end{tabular}

For a continuous space optimisation a fixed architecture is chosen with three parameters open to variations within a fixed upper and lower bound. Figure 24 shows these parametric values, in terms of the GA population pool averages, as the iterations advance. With continuous crossbreeding and mutation, values with high competitiveness are increasingly inherited and finally converge as no new outcompeting alternatives are discovered. For an MTOW minimisation, the resulting parameters are trivial and merely represent an optimisation test scenario. Lowest payload mass, highest PV efficiency and lowest cruise and loiter altitude are established after termination for all repeated optimisation runs. Results for the case study B1, optimising the discrete concept and five continuous parameter space for two objectives are shown in Figure 25. Over the complete iterations all resulting objective 
values of evaluated concepts are shown, together with the resulting Pareto-front. Results are also categorised according to their base power provision technologies, to highlight the clear discontinuity of the resulting front. The three main regions result from the differences in the pure battery, SO-FC and URPEM-FC power systems, as they compete with the given parameter combinations. All Proton exchange membrane fuel cell (PEM-FC) concepts are dominated, as they are outcompeted by the SO-FC concepts in terms of architecture mass. Other URPEM-FC and pure battery concepts compete along the front, and provide the tradeoff between take-off mass and subsystem complexity.
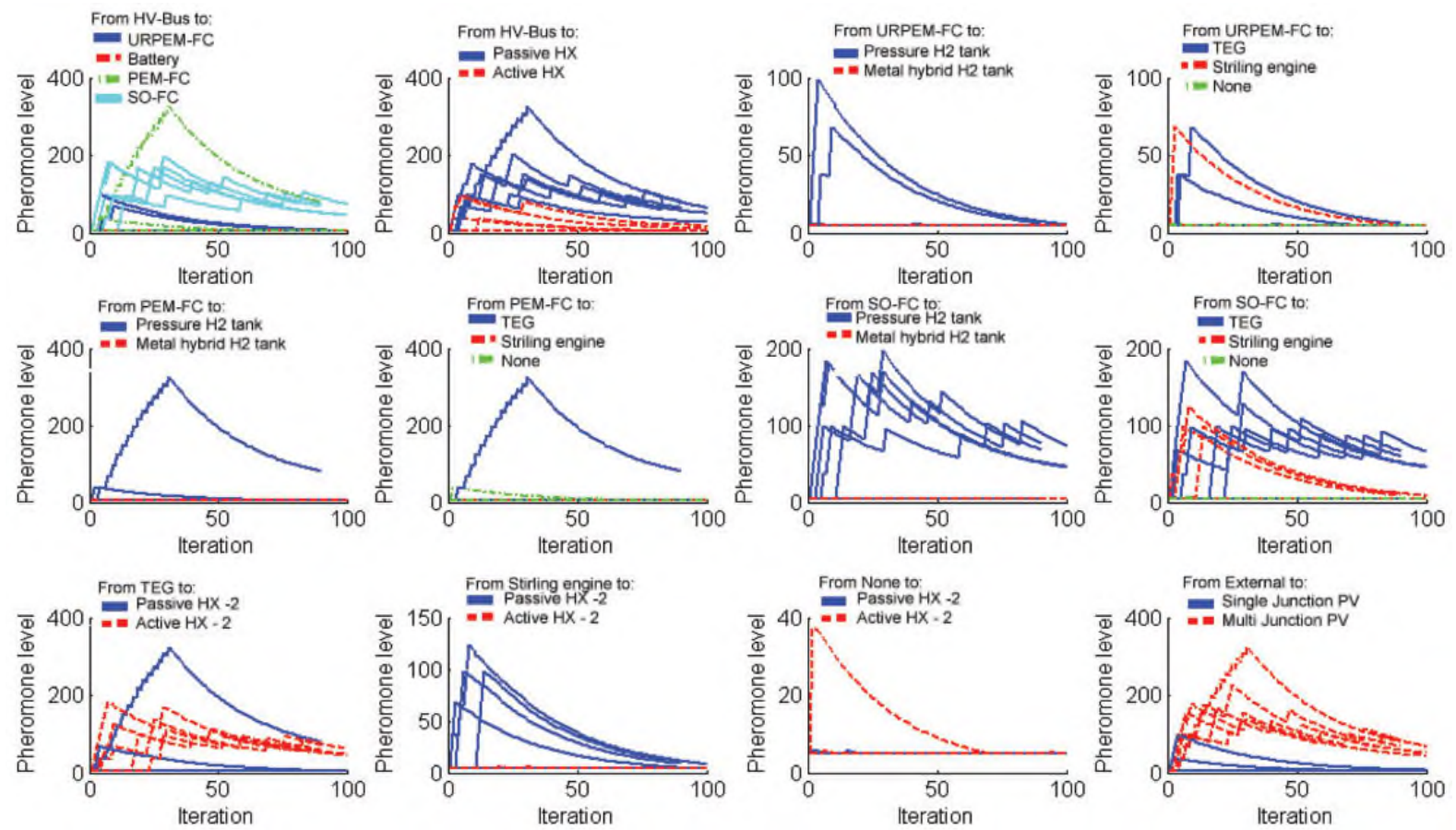

Figure 23: ACO Pheromone value evolution - Case A1
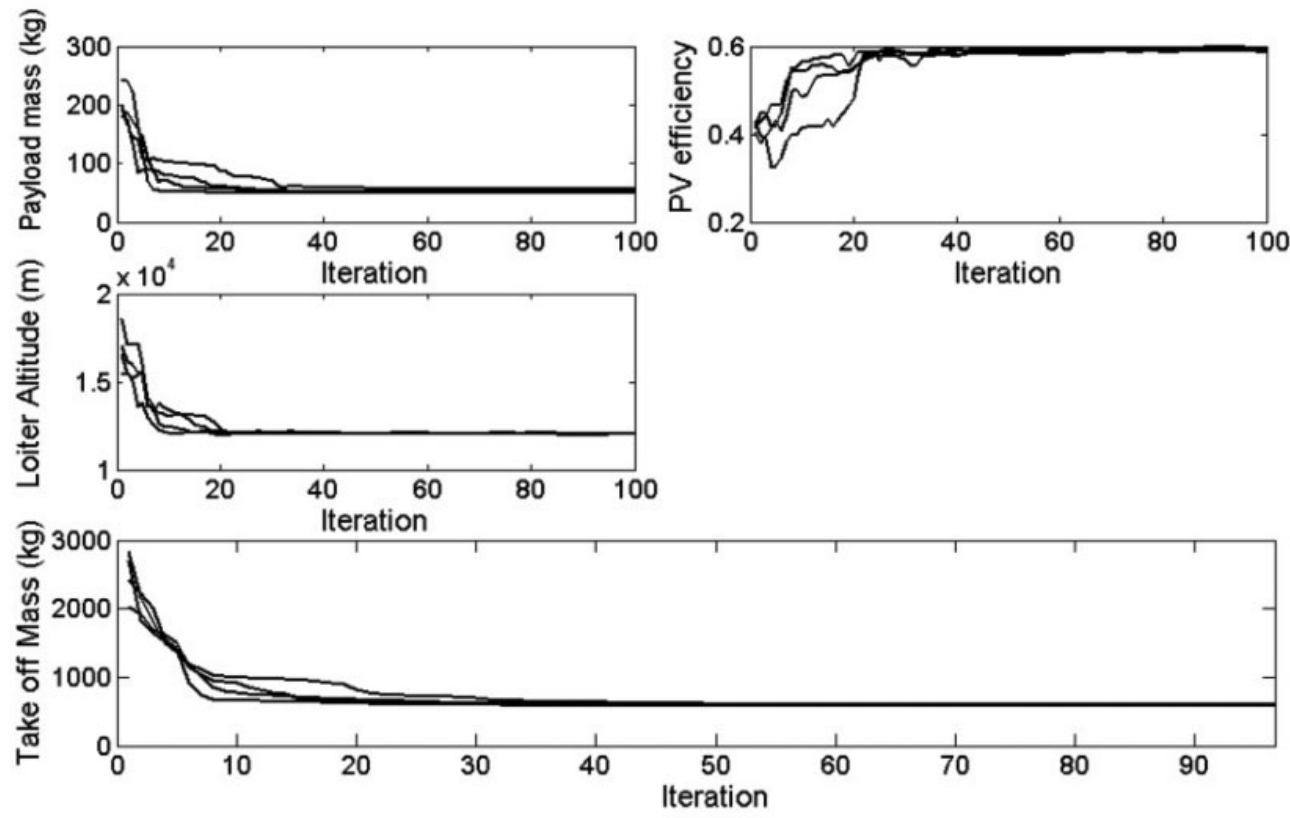

Figure 24: Parameter convergence of GA population pool - Case A2 
The presented results from enumeration to parameter sweeps and optimisation studies lead to the following technical design recommendations. Utilising an SO-FC for the main power provision is most beneficial in terms of architecture mass, if combined with a TEG waste heat component, while retaining a relatively low complexity value, compared to other competitors. Including continuous design parameters in the analysis, does not yield a radical change in competitive concepts. SO-FC still dominate in the architecture mass direction, but pure battery concepts now also emerge with a much lower complexity value. As a good trade-off between the two the URPEM-FC may be chosen.

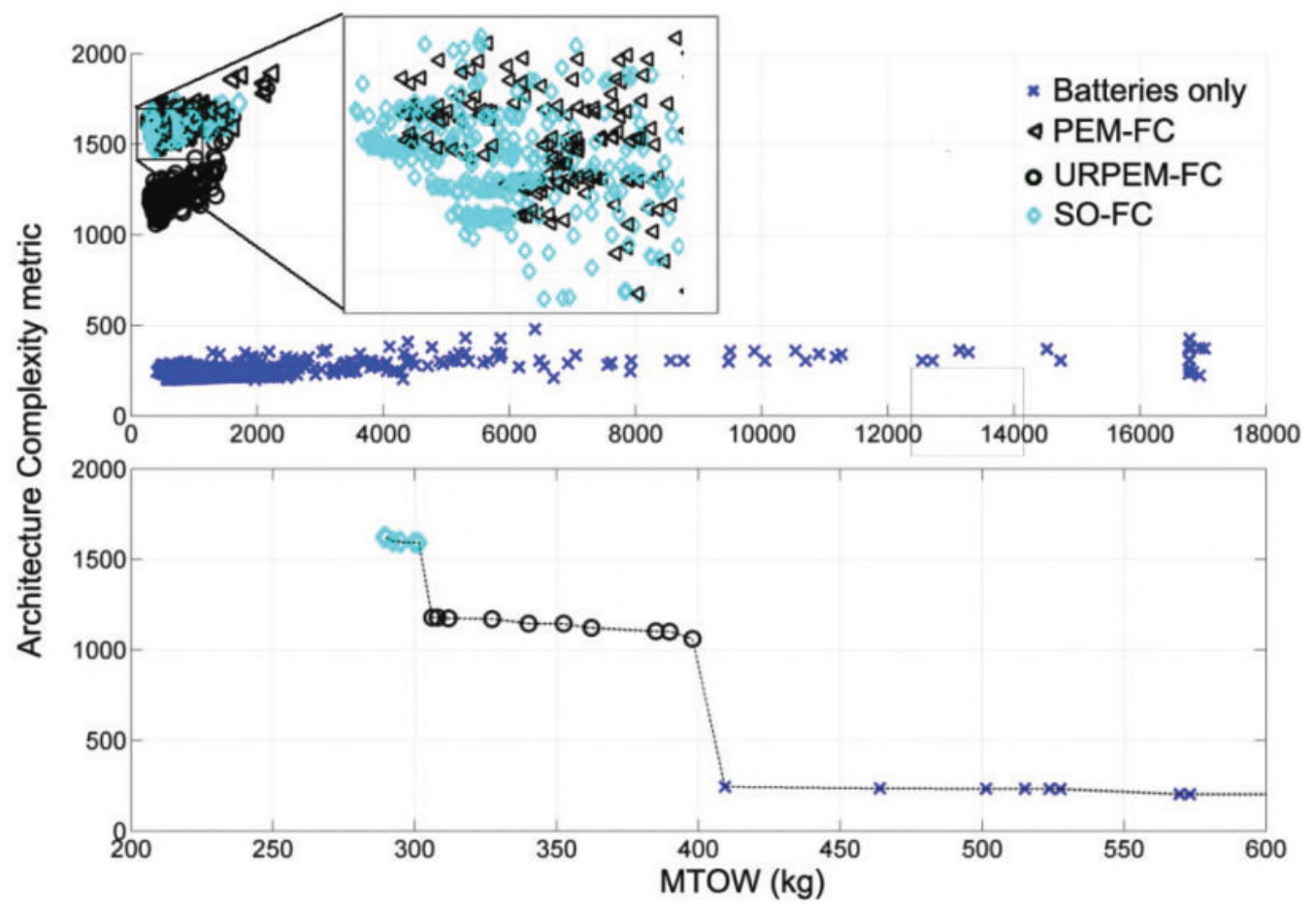

Figure 25: Analyse concept and resulting Pareto-front - Case B1

\section{Conclusions}

A highly automated approach to conceptual aircraft subsystem design has been presented. The population of a central database of design information and the effective combination of individual mathematical models of system components leads to automated generation and analysis of system architectures. The process was demonstrated for a design study of the power systems of an energy balanced UAV operating at high altitudes and long endurances. Rudimentary component models were considered, to verify the correct operation of the interrelated automated processes. Selection is then enabled by two main options. Designers can either use the full enumeration of the architecture concept space, coupled with parameter sweeps and design objective weighting to establish a subset of competitive architectures. Alternatively they can conduct a full multi-objective optimisation with the new defined hybrid optimiser to establish the Pareto-fronts. Such flexibility in decision making and broader investigation of the design space contributes to the current pool of design methods and extends their capabilities.

The application study shows some of the benefits of adopting such a design approach. Trade-off diagrams for UAV power systems architectures show the performance impact of design choices for architectural composition and design problem parameters. This provision to investigate variability of both component technologies and design values with a single framework makes comparisons much more compelling. Architectures of vastly different 
nature and their results can now be handled efficiently, eliminating the need for recalibration of method dependent data for different architectures. Results specific to the UAV investigation show a generally superior performance in MTOW and power ratio for SO-FC architectures. Once also continuous parameters were considered, such as loiter altitude and battery specific energy, a range of architecture types emerged as competitive along the Pareto front for a MTOW and complexity trade-off. In addition the methods useful place within the overall aircraft systems design process has been discussed.

Future work will focus on improvements to the component models for the UAV case study and an extension of the framework to new case studies. In addition, optimiser parameter settings and their impact on convergence accuracy and rate, require further in-depth study.

\section{References}

1. Raymer DP. Aircraft aesign: A conceptual approach. 4th ed. AIAA Education Series. Reston, VA: AIAA, 2006.

2. Howe D. Aircraft conceptual design synthesis. London: Professional Engineering Publishing Limited, 2000.

3. Mattingly D, Heiser WH and Pratt DT. Aircraft engine design. 2nd ed. AIAA Education Series. Reston, VA: AIAA, 2003.

4. Moir I and Seabridge A. Design and development of aircraft systems: An introduction. 2nd ed. London: Professional Engineering Publishing Limited, 2012.

5. Moir I and Seabridge A. Aircraft systems: Mechanical, electrical, and avionics subsystems integration. 3rd ed. New York: Wiley, 2011.

6. Mavris D, Tenorio C and Armstrong M. A methodology for aircraft systems architecture definition. In: 46th AIAA aerospace sciences meeting, Reno, Nevada, 7-10 January 2008.

7. Mavris D, Tenorio $C$ and Armstrong $M$. Architecture subsystem sizing and coordinated optimization methods. In: 47th AIAA aerospace sciences meeting, Orlando, FL, USA, January 2009.

8. Armstrong M. A process for function based architecture definition and modeling. MSc Thesis, Georgia Institute of Technology, USA, 2008.

9. Judt DM and Lawson CP. Methodology for automated aircraft systems architecture enumeration and analysis. In: AIAA ATIO Conference, Indianapolis, Indiana, USA, 2012.

10. Liscouet-Hanke S. A simulation framework for aircraft power systems architecting. In: ICAS, Anchorage, Alaska, 2008.

11. Liscouet-Hanke S. A model-based methodology for integrated preliminary sizing and analysis of aircraft power system architectures. PhD Thesis, Universite' de Toulouse, 2008.

12. Tenorio C. Methods for collaborative conceptual design of aircraft power architectures. PhD Thesis, Georgia Institute of Technology, USA, 2010.

13. Olvander J, Lunden B and Gavel H. A computerized optimization framework for the morphological matrix applied to aircraft conceptual design. Comput-Aid Des 2009; 41: 187196.

14. Gavel H, Oelvander J, Johansson B, et al. Aircraft fuel system synthesis aided by interactive morphology and optimization. In: 45th AIAA Aerospace sciences meeting, Reno, Nevada, 2007.

15. Safavi E, Gopinath V, Olvander J, et al. A collaborative tool for conceptual aircraft systems design. In: AIAA Modeling and simulation technologies conference, Minneapolis, Minnesota, 2012.

16. Chepko A, Weck O, Linne D, et al. Architecture modeling of in-situ oxygen production and its impacts on lunar campaigns. In: AIAA SPACE 2008 conference \& exposition, San Diego, California, 2008.

17. Pate $D$, Patterson $M$ and German $B$. Methods for optimizing a family of reconfigurable aircraft. In: 11th AIAA Aviation technology, integration and operations, Virginia Beach, VA, USA, 2022 September 2011. 
18. Villeneuve F. A method for concept and technology exploration of aerospace architectures. PhD Thesis, Georgia Institute of Technology, USA, 2007.

19. Zeidner L, Rock B, Desai N, et al. Application of a technology screening methodology for rotorcraft alternative power systems. In: 48th AIAA Aerospace sciences meeting, Orlando, Florida, 2010.

20. Dorigo M and Gambardella LM. Ant colonies for the traveling salesman problem. Biosystems 1997; 43(2): 73-81.

21. Judt DM. Methods for automated aircraft systems architecture generation, analysis and selection. PhD Thesis, Cranfield University, UK, 2014.

22. SAE International. Guidelines and methods for conducting the safety assessment process on civil airborne systems and equipment. Aerospace Recommended Practice 4761, Issued 1996 $-12,1996$.

23. SAE International. Certification considerations for highly-integrated or complex aircraft systems. Aerospace Recommended Practice 4754, Issued 1996 -11, 1996.

24. INCOSE. Systems engineering handbook v.3, 2006.

25. International Standard. Systems and software engineering - Systems life cycle processes. ISO/IEC 15288, 2008.

26. Moir I and Seabridge A. Design and development of aircraft system. 2nd ed. New York: Wiley, 2013.

27. HZ-20 thermoelectric module datasheet. San Diego, CA: Hi-Z Technology, Inc., 2002.

28. Fleming J, Ng W and Ghamaty S. Thermoelectric power generation for UAV applications. In: 1st International energy conversion engineering conference, Portsmouth, Virginia, 2003.

29. Navid A, Vanderpool D, Bah A, et al. Towards optimization of a pyroelectric energy converter for harvesting waste heat. Int J Heat Mass Transfer 2010; 53: 4060-4070.

30. Stirling engine assessment. Palo Alto, CA: EPRI, 2002.

31. Wood JG and Lane N. Advanced $35 \mathrm{~W}$ free-piston stirling engine for space power applications. Space Technology and Applications International Forum- STAIF, Albuquerque, New Mexico, 2003.

32. Geankoplis CJ. Transport processes and separation process principles. 4th ed. Englewood Cliffs, NJ: Prentice Hall, 2003.

33. Spiegel C. PEM fuel cell modeling and simulation using MATLAB. 1st ed. San Diego, CA: Academic Press, 2008.

34. Xiaojin L, Yu X, Zhigang S, et al. Mass minimization of a discrete regenerative fuel cell (RFC) system for onboard energy storage. J Power Sources 2010; 195: 4811-4815.

35. Federal Aviation Regulations, Part 25: Airworthiness standards: Transport category.

36. Lapena-Rey N, Mosquera J, Bataller E, et al. First fuelcell manned aircraft. J Aircraft 2010; 47(6): 1825-1835.

37. Romeo G, Moraglio I and Novarese C. ENFICA-FC: Preliminary survey \& design of 2-seat aircraft powered by fuel cells electric propulsion. In: 7th AIAA Aviation technology, integration and operations conference, Belfast, Northern Ireland, 18-20 September 2007.

38. Ross H. Fly around the world with a solar powered airplane. In: 26th Congress of international council of the aeronautical sciences (ICAS), Anchorage, Alaska, 14-19 September 2008.

39. http://www.solarimpulse.com/en/airplane (accessed December 2013).

40. Richardson TD. Phantom eye - accelerated air vehicle structural development through prototyping. In" 53rd AIAA/ASME/ASCE/AHS/ASC Structures, structural dynamics and materials conference, Honolulu, Hawaii, 2012

41. http://www.avinc.com/uas/stratospheric/global observer//accessed March 2014)

42. Sachs $G$, Lenz $J$ and Holzapfel $F$. Unlimited endurance performance of solar UAVs with minimal or zero electric energy storage. In: AIAA Guidance navigation and control conference, Chicago, Illinois, 2009.

43. Montagnier $\mathrm{O}$ and Bovet $\mathrm{L}$. Optimization of a solarpowered high altitude long endurance UAV. In: 27th International congress of the aeronautical sciences, Nice, France, 2010. 
2014-11-19

\section{Application of an automated aircraft architecture generation and analysis tool to unmanned aerial vehicle subsystem design}

Judt, David M.

SAGE Publications

Judt DM \& Lawson CP (2015) Application of an automated aircraft architecture generation and analysis tool to unmanned aerial vehicle subsystem design, Proceedings of the Institution of Mechanical Engineers, Part G: Journal of Aerospace Engineering, Volume 229, Issue 9, pp.1690-1708. https://doi.org/10.1177/0954410014558691

Downloaded from Cranfield Library Services E-Repository 